Apidologie 1980, 11 (2), 113-130.

\title{
MORPHOLOGIE DER ANTENNENPUTZAPPARATE BEI APOIDEA
}

\author{
Morphologie de l'appareil de nettoyage des antennes \\ chez les Apoüdes
}

\author{
Klaus SCHÖNITZER und Maximilian RENNER \\ Zoologisches Institut der Universität, Luisenstr. 14, D-8000 München 2
}

\section{SUMMARY}

MORPHOLOGY OF THE ANTENNA CLEANER IN APOIDEA

The morphology of the tibio-tarsal antenna cleaner is described using SEM preparations. The phylogeny and the systematic relevance of the antenna cleaner is discussed.

\section{ZUSAMMENFASSUNG}

Die Morphologie der tibio-tarsalen Putzapparate am Vorderbein verschiedener Apoidea wird aufgrund rasterelektronenmikroskopischer Untersuchungen beschrieben. Die Phylogenie der Putzapparate sowie Übereinstimmungen zwischen ihrem Bau und dem System der Apoidea werden diskutiert.

\section{EINLEITUNG}

Spezialisierungen der Vorderbeine, die dem Reinigen der Antennen dienen, sind bei verschiedenen Insektenordnungen bekannt, z. B. bei Rhynchoten, Coleopteren, Lepidopteren und Hymenopteren (Poessinger 1948, Regenfuss 1975, Weber 1933). Die Putzapparate der Bienen wurden bereits durch DE GEER 1752/76, CANESTRINI und Berlese 1880, KIRBY 1802 und DAHL 1884 beschrieben. Die eingehendste Untersuchung stammt von GENNERICH 1922. Weitere Beschreibungen bzw. 


\section{Zeichnungen von Putzapparaten bei Apoidea findet man bei ALFORD 1975, BEEKEN 1934, Eickwort 1969, Michener 1944, SNodgrass 1956 und Wagner 1959.}

In der vorliegenden Arbeit wird die Morphologie der Putzapparate verschiedener Apoidea aufgrund rasterelektronenmikroskopischer Untersuchung beschrieben. Die Phylogenie der Putzapparate und ihr möglicher systematischer Wert werden diskutiert.

\section{MATERIAL UND METHODE}

Untersuchte Bienen (Systematik nach MiCHENER 1974) : Colletidae : Colletes succinctus L. (1우); Andrenidae : Andrena ovulata KIRBY (19); Halictidae: Halictus sexcinctus F. (19); Melittidae : Melitta leporina Panz. (1 3 .), Dasypoda argentata PANz. (1 9 ); Megachilidae : Anthidium septemdentatum Latr.

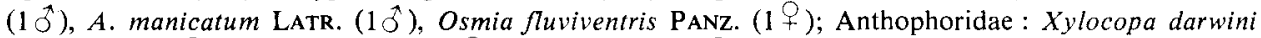

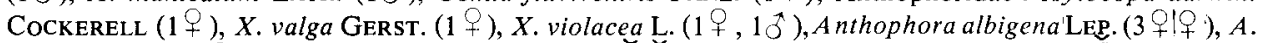

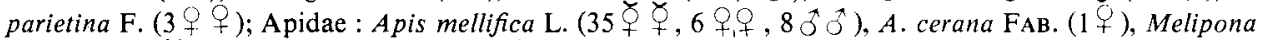
scutellaris (1ఫ), Bombus agrorum F. $(2 q+, 10), B$. hortorum L. (1q), B. hypnorum L. (1 $q), B$.

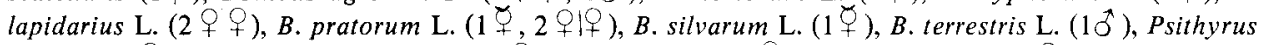
lucorum (1 + ), P. meridionalis RichDs. (1 + ), P. rupestris F. (1 + ), P. silvestris LeP. (1 9 ), Euglossa spec. (19).

Die meisten Präparate wurden von genadelten Tieren gemacht, Vergleichspräparate auch von frisch gefangenen Honigbienen und Hummeln sowie von Alkoholmaterial. Es ergaben sich hierbei keine Unterschiede.

Durch die kräftige, chitinöse Beschaffenheit der Putzapparate erübrigt sich eine besondere Fixierung. Die Beine wurden nach einer Reinigung im Ultraschall besputtert und im Cambridge Stereoscan gerastert. Bezüglich der Orientierung der Beine siehe Abb. 1 : Ansicht der Ventralseite des linken Vorderbeines - die dorsale Seite des Beines liegt unter der Zeichenebene. Die Abbildungen der Putzapparate sind so orientert, dass distal oben und proximal unten ist. Stets sind linke Beine abgebildet. Der beigefügte Massstab entspricht 0,5 mm. Punktierte Linien geben die Grenzen der Behaarung an; gestrichelt sind die Konturen der Beine, soweit ihr genauer Verlauf durch Haare verdeckt ist. Der Putzapparat der Bienen befindet sich ventral im Bereich des Gelenks zwischen Tibia und Basitarsus. Er besteht aus einem Sporn, der am distalen Ende der Tibia beweglich eingelenkt ist, und einer im proximalen Teil des Basitarsus gelegenen etwa halbzylindrischen Einbuchtung ("Scharte »), an deren hinterem Ende ein mehr oder weniger

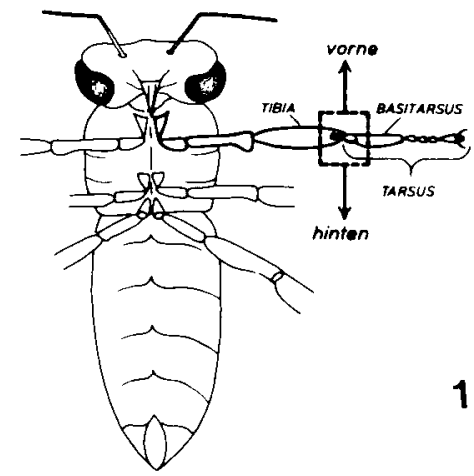

ABB. 1. - Biene, schematisch, auf dem Rücken liegend.

Das strichlierte Viereck entspricht dem Bildausschnitt der Abb. 3.

FIG. 1. - Abeille, vue schématique dorsale.

Le carré en pointillés correspond à la Fig. 3 . 
halbkreisförmiger Kamm steht. Der Sporn ist beweglich inseriert und kann als ganzes nach ventral (vom Basitarsus weg) bis zum Anschlag an der distal-ventralen Kante der Tibia (Abb. 2-7) bewegt werden, obwohl weder an seiner Basis noch sonstwo Muskeln ansetzen (GENNERICH 1922, SNODGRAsS 1956). In Ruhestellung ist er mit seiner Spitze distad gerichtet. Die Beine wurden in geringfügig unterschiedlich gestrecktem Zustand betrachtet, wodurch in den einzelnen Bildern die relative Stellung zwischen Sporn und Scharte etwas variiert, zumal sich der Sporn auch um seine Längsachse etwas drehen lässt. Das Verhältnis aus der Länge des Spornes zu seiner grössten Breite wurde als "Spornindex" definiert, entsprechend der Quotient der Öffnungsweite der Scharte zu ihrer Tiefe als "Schartenindex ". Die Messstrecken wurden aus Aufnahmen wie sie Abb. 8 und 9 zeigen, gemessen.
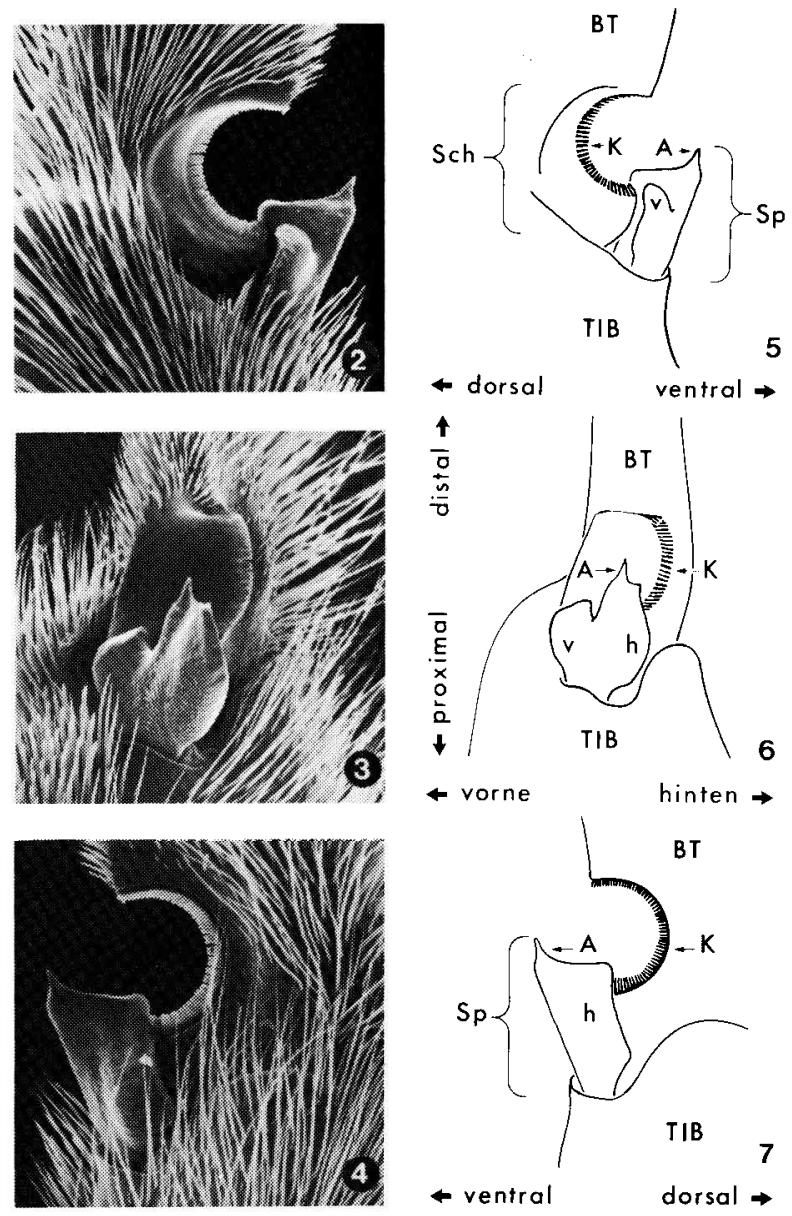

Aв8. 2-7. - Putzapparat einer Apis mellifica carnica $P$ von vorne (Abb. 2 und 5), von ventral ( $A b b .3$ und 6) und von hinten ( $A b b .4$ und 7 ).

Die Aufnahmen sind vom selben Objekt, das jeweils um $90^{\circ}$ gedreht wurde.

Vergrösserung ca. 80 fach. $\mathrm{BT}=$ Basitarsus, $\mathrm{TIB}=$ Tibia, $\mathrm{A}=\mathrm{Apex}$,

$\mathrm{K}=\mathrm{Kamm}, \mathrm{Sch}=$ Scharte, $\mathrm{Sp}=\mathrm{Sporn}, \mathrm{h}=$ hinteres Velum, $\mathrm{v}=$ vorderes Velum.

FIG. 2-7. - Appareil de nettoyage d'une $f$ d'Apis mellifica carnica. Vue antérieure (Fig. 2 et 5), ventrale (Fig. 3 et 6 ) et postérieure (Fig. 4 et 7).

Les clichés sont du même objet que l'on a fait pivoter à chaque fois de $90^{\circ}$. Grossissement environ $\times 80$.

$\mathrm{BT}=$ basitarse; $\mathrm{TIB}=$ tibia $; \mathrm{A}=$ apex $\mathrm{K}=$ peigne; $\mathrm{Sch}=$ brèche;

$\mathrm{S} \mathrm{p}=$ éperon; $\mathrm{h}=$ velum postérieur; $\mathrm{v}=$ velum antérieur. 


\title{
BESCHREIBUNG DER UNTERSUCHTEN PUTZAPPARATE
}

\author{
Apis mellifica
}

Der Sporn besteht aus einem Stamm (z.T. " Malus " genannt) und zwei unterschiedlich grossen Vela. Der Stamm ist gerade und erscheint im Lichtmikroskop dunkel. Das grössere der beiden Vela ist schräg nach hinten - dorsal gerichtet; es ist ein dünner Lappen, der im Lichtmikroskop durchscheinend hell ist, sich im Rasterelektronenmikroskop jedoch nicht immer klar vom Stamm abgrenzen lässt. Das zweite Velum ist daumenartig nach vorne - dorsal gerichtet, und ist etwa ebenso dick wie der Stamm. Sein distaler Teil bildet in der Regel einen leicht dorsad gebogenen Vorsprung. Die beiden Vela stehen zueinander in einem Winkel von ca. $70^{\circ}$ bis $100^{\circ}$. Die sich über das hintere Velum erhebende Spitze des Stammes nennen wir “ Apex ». Seine anteilmässige Länge am gesamten Sporn beträgt bei $A$. mellifica $1 / 4$ bis $1 / 5$. Die Oberfläche des Spornes ist unterschiedlich strukturiert, am stärksten ist der Stamm, insbesondere an seiner Basis " beschuppt ", und zwar in der gleichen Weise wie die nicht behaarten Teile des Tarsus und der Tibia (Abb. 2 bis 4;8). Die beiden Vela haben ventral bei den einzelnen Tieren eine fast vollkommen glatte Oberfläche, die zur Scharte gewandte dorsale Seite des Spornes ist in der Regel vollkommen unstrukturiert. Die Struktur der Oberfläche des Spornes variiert von Individuum zu Individuum beträchtlich.

Die Putzscharte ist eine annähernd halbzylindrische Einbuchtung des proximalen Teils des Basitarsus, die von hinten gesehen etwa halbkreisförmig ist. Sie trägt an ihrem hinteren Rand den Kamm. Nach vorne erweitert sie sich etwas und ist leicht nach proximal gerichtet (Abb. 2 und 3). Die Innenfläche der Scharte ist bei den meisten Individuen höchstens im gelenknahen (proximalen) Teil gering strukturiert. Der grösste Teil ist in der Regel vollständig glatt. Der Kamm besteht aus einer Reihe von Zähnen, die nach hinten gerichtet und etwas zur Mitte der Ausbuchtung gebogen sind. Dadurch wird die Scharte konisch

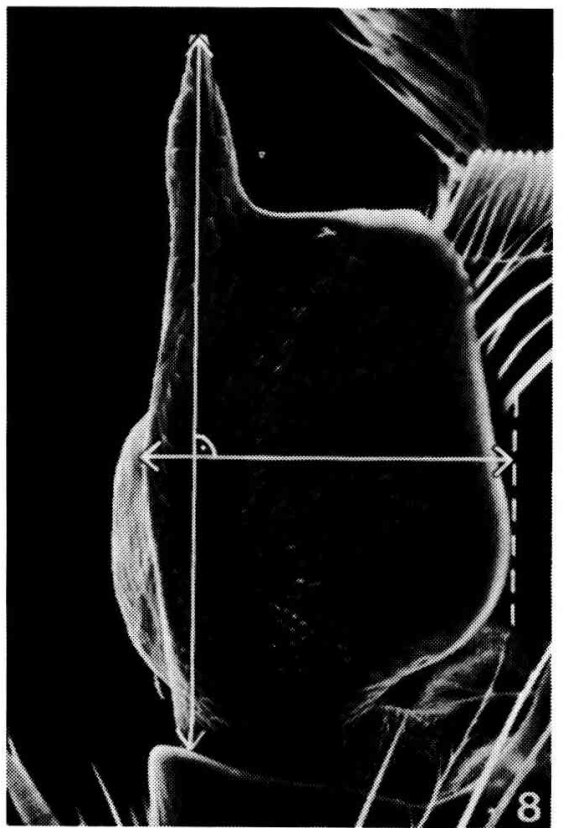

Авв. 8. - Sporn einer Apis mellifica carnica 9 von hinten gesehen, senkrecht auf das hintere Velum. Die Pfeile kennzeichnen die Messstrecken für die Berechnung des Spornindex. Ca. 190 fach vergrössert.

FIG. - Éperon d'une $q$ d'Apis mellifica carnica.

Vue postérieure, perpendiculairement au velum postérieur.

Les flèches indiquent les mesures prises pour le calcul de l'indice de l'éperon. $190 \times$ environ. 
verengt. Die Zähne sind 32 bis $45 \mu \mathrm{m}$ lang, ihre Breite schwankt zwischen 4,5 und $8,0 \mu \mathrm{m}$. Der Kamm ist gegenüber der hinteren Fläche des Basitarsus durch eine Rinne abgesetzt, die sich proximal etwas erweitert (Abb. 9 und 10). Die zur Rinne gerichtete Seite der Zähne ist glatt, während die gegenüberliegende Seite, die die Scharteninnenfläche fortsetzt, etwa von der Mitte an bis zu ihrer Spitze gerieft ist (Abb. 10 bis 12). Die Rinne sowie die Form und Struktur des Kammes waren bei allen untersuchten Apoidea nahezu gleich.

Im folgenden wird auf die Scharten nur eingegangen, soweit sie sich deutlich von denen bei $A$. mellifica unterscheiden.
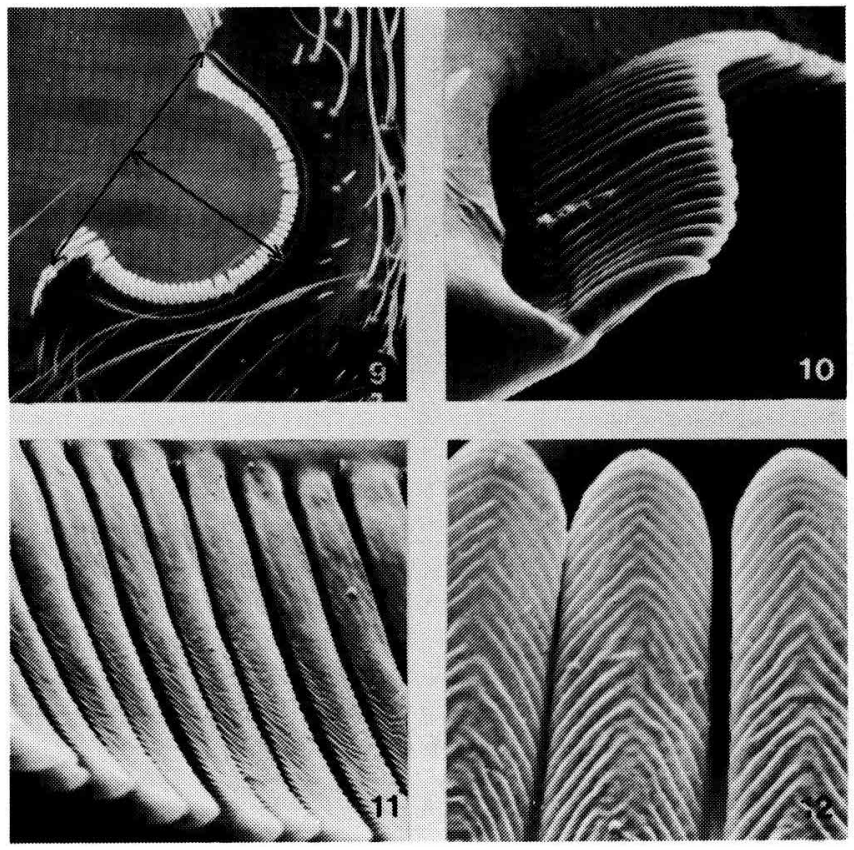

ABв. 9. - Scharte einer Apis mellifica 9 von hinten.

Senkrecht auf die Kammebene gesehen, ähnliche Blickrichtung wie in Abb. 4.

Die Pfeile kennzeichnen die Messtrecken für die Berechnung des Schartenindex. Ca. 95 fach.

FIG. 9. - Brèche d'une $\not$ d'Apis mellifica. Vue postérieure, perpendiculairement à la surface du peigne, direction de vue comme dans la Fig. 4. Les flèches indiquent

les mesures prises pour le calcul de l'indice de la brèche. $95 \times$ environ.

Авв. 10. - Distaler Teil des Kammes mit Rinne einer Melipona scutellaris $\not{q}$.

Blickrichtung von ventral, ähnlich wie in Abb. 3. Ca. 390 fach.

FIG. 10. - Partie distale du peigne avec la gouttière, d'une $\not{\zeta}$ de Melipona scutellaris.

Direction de vue ventrale comme dans la Fig. 3. $390 \times$ environ.

Aвв. 11. - Kamm von Psithyrus rupestris $q$, von hinten - ventral gesehen.

Am Bildoberrand ein Teil der (glatten) Scharteninnenfläche. Ca. 800 fach.

FIG. 11. - Peigne d'une $O$ de Psithyrus rupestris. Vue postéro-ventrale.

A la limite supérieure de la photo une partie de la surface intérieure de la brèche (lisse). $800 \times$ environ.

AвB. 12. - Spitzen der Kammhaare bei P. rupestris $q$, von ventral, etwa senkrecht auf den Kamm gesehen. Ca. 2500 fach.

FIG. 12. - Pointe des dents du peigne chez une $q$ de P. rupestris.

Vue ventrale, à peu près perpendiculaire au peigne. $2500 \times$ environ. 
Apis cerana

Der Putzapparat der Apis cerana gleicht dem von A. mellifica völlig, er ist lediglich - entsprechend der Körpergrösse - kleiner.

\section{Melipona}

Der Putzapparat von Melipona scutellaris hat im Gegensatz zu denen von Apis nur ein, nach hinten - dorsal gerichtetes Velum. Es ist an seinem distalen Rand leicht verdickt (Abb. 14, 15; schwarzer Pfeil). Der Apex - ca. 1/5 der Spornlänge - ist spitzer als bei Apis. Der Stamm des Spornes hat vorne eine leichte Ausbuchtung (Abb. 14, weisser Pfeil).

\section{Bombus und Psithyrus}

Die untersuchten Bombini haben einen Putzapparat desselben Typs (Abb. 16-18). Der Sporn trägt ein Velum - entsprechend dem grösseren von A pis - sowie einen Vorsprung an der Vorderseite des Stammes, der dem kleineren Velum von $A$ pis entspricht. Die schuppige Oberflächenstrukturierung ist am Rücken des Stammes besonders deutlich. Etwa in seiner Mitte gehen die spitzen Schuppen in eine Zähnchenreihe über, die fast bis zur Spitze des Apex reicht (Abb. 16-18 $\rightarrow$ ). Eine zweite, dorsade Zähnchenreihe (Abb. 17 $18<$ ) befindet sich an der gegenüberliegenden (hinteren) Kante des Apex. Dieser ist länger (ca. 1/3 der Gesamtlänge des Spornes) und spitzer als bei Apis.

\section{Euglossa}

Der Sporn der Euglossa spec. (Abb. 19-21) hat ein deutliches zweites (vorderes) Velum, ähnlich dem von Apis (Pfeil in Abb. 19, 20). Das hintere grössere Velum ist an seinem oberen, distalen Rand verdickt. Eine derartige Verdickung nennt GeNNERICH (1922) "Seitenast des Stammes "; sie ist - ebenso wie der Stamm - im Lichtmikroskop dunkel im Gegensatz zur durchscheinend hellen Fläche des Velums. Die apical-hintere Ecke des Velums bildet mit diesem Seitenast des Stammes ein spitzes Dreieck. Wie bei den Hummeln trägt der Sporn zwei Zahnreihen, jedoch mit grösseren Zähnen. Der leicht nach aussen (ventrad) gebogene Apex misst ca. 1/3 der gesamten Spornlänge.

\section{Xylocopa}

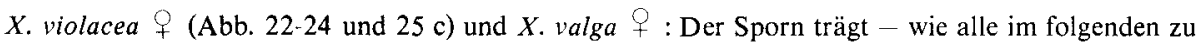
besprechenden - nur ein nach hinten-dorsal gerichtetes Velum. Der Stamm ist, ähnlich wie bei Melipona, vorne leicht ausgebuchtet und trägt zwei Zahnreihen. Eine Verdickung am distalen Rand des Velums (Steitenast des Stammes) ist angedeutet. Der Apex nimmt ca. 3/8 der gesamten Spornlänge ein.

$X$. violacea $\sigma^{\star}$ (Abb. 25 b) : Das Velum ist gross and fast quadratisch. Der dünne Apex misst nur etwa 1/6 der Spornlänge. Die dorsal - hintere Zahrreihe bewehrt die Hälfte der distalen Kante des Velums.

$X$. darwini $f$ (Abb. 25 a): Das auffallend kleine Velum ist an einem Seitenast des Stammes aufgehängt (Pfeil), der sich hinten gabelt. Der Apex bildet die Hälfte der gesamten Spornlänge und trägt die hintere Zahnreihe.

Die Scharten von $X$. violacea $\hat{\sigma}$ und $q$ sind relativ weit geöffnet (bzw. wenig tief) : Schartenindices 1,8 und 1,9 (vergleiche Tab. 1).

\section{Anthophora}

Charakteristisch ist die distad gerichtete Spitze am Rand des Velums (Abb. 27, 28 Pfeil); Sie ist bei $A$. albigena noch deutlicher als bei $A$. parietina. Von den beiden Zahnreihen hat, insbesondere bei $A$. albigena, die dorsal - hintere etwas längere Zähne. Das Velum ist weit ausladend : man beachte den niederen Spornindex (Tab. 1) trotz des langen Apex (2/5 der Gesamtlänge). Bei A.parietina befinden sich an der Aussenseite des Velums kleine Haare. Bei $A$. albigena trägt der Sporn, vor allem das Velum, spitze Schuppen. Die Scharte von A. albigena ist relativ weit geöffnet (Tab. 1). 


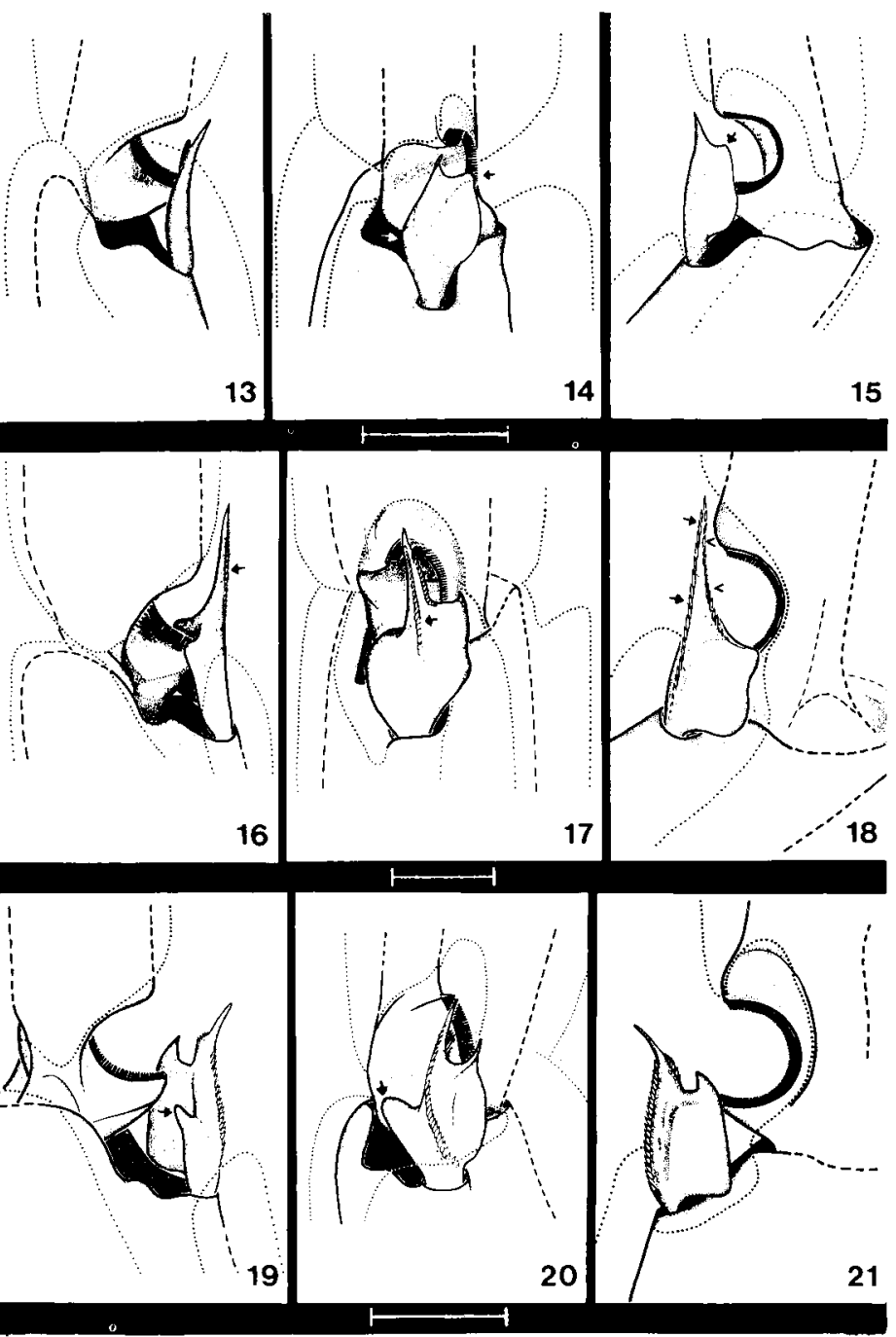

Aвв. 13-15. - Putzapparat einer Melipona scutellaris $\not$ von vorne (13), von ventral (14) und ron hinten (15).

FIG. 13-15. - Appareil de nettoyage d'une $\&$ de Melipona scutellaris. Vue antérieure (Fig. 13), ventrale (Fig. 14) et postérieure (Fig. 15).

ABB. 16-18. - Putzapparat von Bombus agrorum + ron vorne (16), von ventral (17) und von hinten (18).

Fig. 16-18. - Appareil de nettoyage d'une 9 de Bombus agrorum.

Vue antérieure (Fig. 16), ventrale (Fig. 17) et postérieure (Fig. 18).

Aвв. 19-21. - Putzapparat einer Euglossa spec. O ron rorne (19), von ventral (20) und ion hinten (21).

Fig. 19-21. - Appareil de netloyage d'une + d'Euglossa sp.

Vue antérieure (Fig. 19), ventrale (Fig. 20) et postérieure (Fig. 21). 

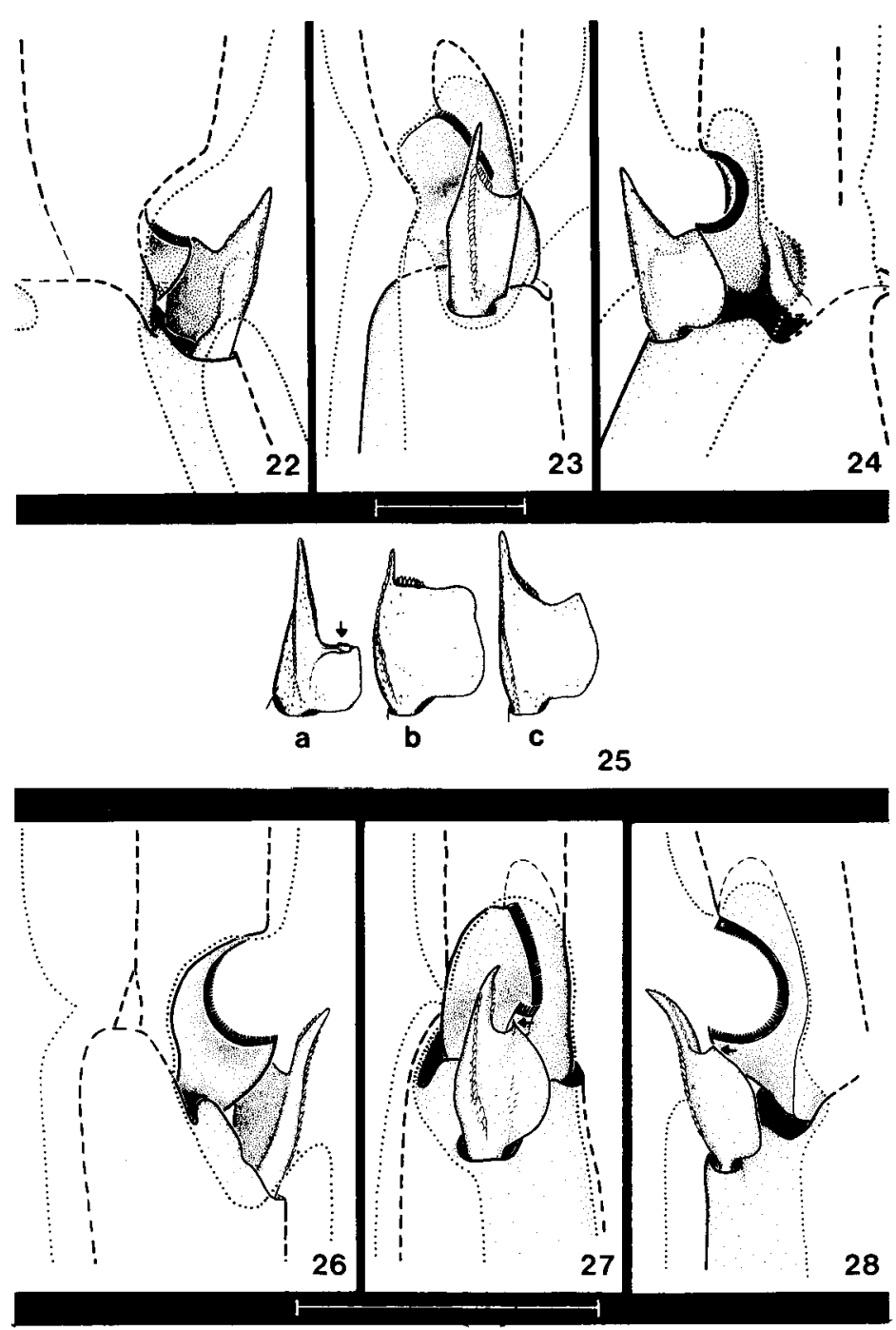

Aвв. 22-24. - Putzapparat einer Xylocopa violacea 9 von vorne (22), von ventral (23) und von hinten (24).

FIG. 22-24. - Appareil de nettoyage d'une $O$ de Xylocopa violacea. Vue antérieure (Fig. 22), ventrale (Fig. 23) et postérieure (Fig. 24).

Aвв. 25. - Sporne von Xylocopa darwini $q(a)$, von X. violacea $\hat{\sigma}(b)$ und von X. violacea $q$ (c); jeweils von hinten, senkrecht auf das Velum gesehen.

FIG. 25. - Éperons d'une 9 de Xylocopa darwini (a), d'un $\hat{\delta}$ de X. violacea (b) et d'une + de $\mathrm{X}$. violacea (c). Vues postérieures, perpendiculaires au velum.

ABB. 26-28. - Putzapparat einer Anthophora parietina $q$ von vorne (26), von ventral (27) und von hinten (28).

Fig. 26-28. - Appareil de nettoyage d'une $+d$ 'Anthophora parietina. Vue antérieure (Fig. 26), ventrale (Fig. 27) et postérieure (Fig. 28). 
TAB. 1. - Sporn- und Schartenindices. Es sind jeweils einzelne Messwerte angegeben;

Bei Apis mellifica: Mittelwerte \pm Standardabweichung, sowie Anzahl der Messwerte von verschiedenen Individuen (n).

TABL. 1. - Indices de l'éperon et de la brèche. Il s'agit à chaque fois de mesures individuelles; chez Apis mellifica : moyenne \pm écart-type,

ainsi que le nombre de mesures de divers individus (n).

\begin{tabular}{|c|c|c|}
\hline & $\begin{array}{c}\text { Spornindex } \\
\text { Indice de l'éperon } \\
\end{array}$ & $\begin{array}{c}\text { Schartenindex } \\
\text { Indice de la brèche } \\
\end{array}$ \\
\hline 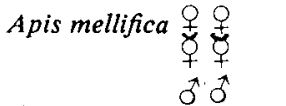 & $\begin{array}{l}2,12 \pm 0,13 ; \mathrm{n}=6 \\
2,29 \pm 0,18 ; \mathrm{n}=9 \\
2,60 \pm 0,12 ; \mathrm{n}=6\end{array}$ & $\begin{array}{l}1,43 \pm 0,05 ; \mathrm{n}=5 \\
1,27 \pm 0,12 ; \mathrm{n}=5 \\
1,53 \pm 0,13 ; \mathrm{n}=6\end{array}$ \\
\hline Apis cerana & 2,5 & 1,4 \\
\hline Psithyrus lucorum & 2,5 & 1,2 \\
\hline P. silvestris & 2,9 & 1,7 \\
\hline P. rupestris & 2,9 & \\
\hline P. meridionalis & 2,5 & 1,5 \\
\hline Bombus silvarum & 2,6 & 1,5 \\
\hline B.terrestris & 2,5 & 1,3 \\
\hline B. pratorum & 2,$3 ; 2,4$ & 1,5 \\
\hline B. lapidarius & 2,3 & 1,5 \\
\hline B. hortorum & 2,5 & 1,3 \\
\hline B. agrorum & 2,$5 ; 2,6 ; 2,6$ & 1,$4 ; 1,4$ \\
\hline Melipona scutellaris & 2,2 & 1,4 \\
\hline Euglossa spec. & 2,1 & 1,2 \\
\hline Xylocopa violacea + & 1,8 & 1,9 \\
\hline & 1,6 & 1,8 \\
\hline Anthophora albigena & 2,$1 ; 2,2 ; 2,2$ & 2,$1 ; 1,9$ \\
\hline A. parietina & 1,$9 ; 2,0$ & 1,$5 ; 1,5$ \\
\hline Osmia fluviventris & 3,0 & 1,9 \\
\hline Anthidium manicatum & 2,1 & 1,6 \\
\hline Dasypoda argentata & 3,9 & 1,7 \\
\hline Melitta leporina & 4,0 & 1,5 \\
\hline Halictus sexcinctus & 3,3 & 2,5 \\
\hline Andrena ovulata & 3,8 & 2,1 \\
\hline Colletes succinctus & 3,8 & 2,8 \\
\hline
\end{tabular}

\section{Anthidium}

(Abb. 29-31). Das breite Velum ist wie bei Euglossa distal verdickt. Die beiden Zahnreihen bestehen aus kräftigen spitzen Zähnen. Der Stamm ist vorne ausgebuchtet, wodurch sich in seiner Mitte ein Vorsprung bildet, der an ein reduziertes Velum erinnert. Vorne und ventral trägt der Stamm spitze, sich dachziegelartig überdeckende Schuppen. Der Apex misst $1 / 3$ bis $1 / 4$ der Gesamtspornlänge. Die Scharte ist am gelenknahen (proximalen) Teil beschuppt und behaart.

\section{Osmia}

(Abb. 32). Der Sporn ist gekennzeichnet durch einen langen Apex (2/5 der Gesamtlänge) und ein schmales Velum (Spornindex 3,0). Der hintere Rand des Velums ist ausgebuchtet. Der Stamm ist wie bei Anthidium beschuppt. Die Scharte ist weit geöffnet bzw. wenig tief (Schartenindex 1,9; Tab. 1).

\section{Dasypoda und Melitta}

Trotz der unterschiedlich starken Behaarung der Beine gleichen sich die Putzapparate weitgehend (Abb. 33-35). Der Sporn hat einen auffällig langen Apex (1/2 der Gesamtlänge) und ein kleines Velum : 
entsprechend hoher Spornindex (3,9 bzw. 4,0). Die Vorderseite des Stammes ist mit spitzen - sich dachziegelartig überdeckenden Schuppen besetzt, die Hinterseite ist nur leicht (narbig) strukturiert. Die ventrale Zahnreihe besteht aus kleineren Zähnen als die dorsale. Der ventroproximale Rand der Scharte trägt Haare und ist zum Teil beschuppt (Abb. 33).
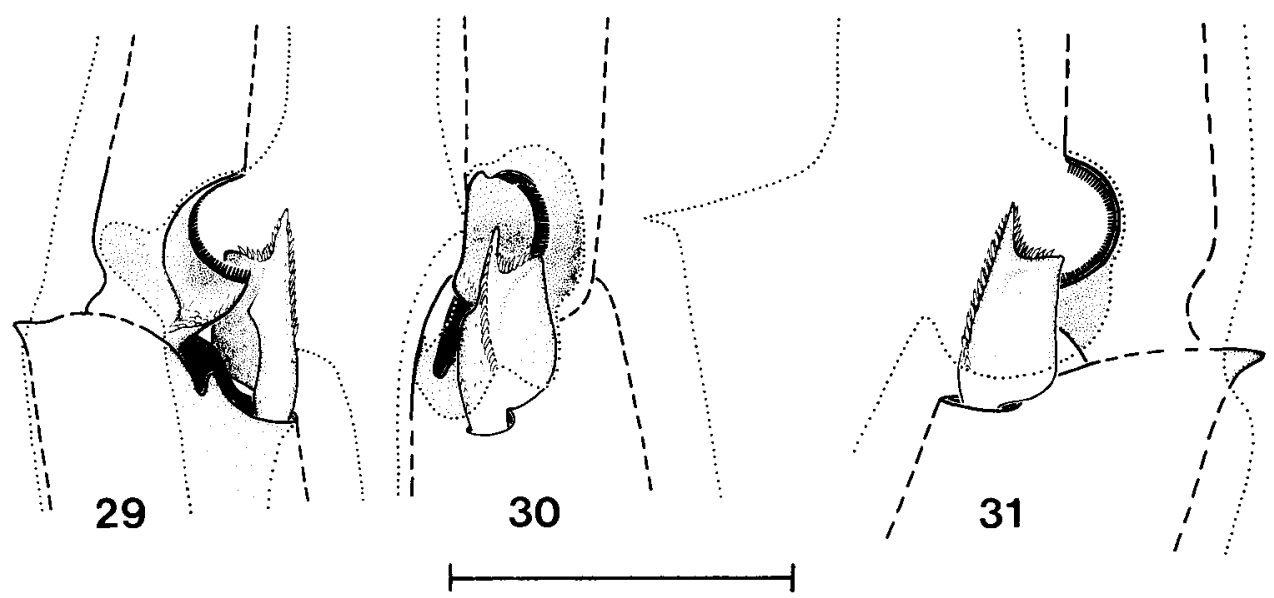

Авв. 29-31. - Putzapparat eines Anthidium manicatum $\widehat{\partial}$, von vorne (29), von ventral (30) ind von hinten (31).

FIG. 29-31. - Appareil de nettoyage d'un dैA d'Anthidium manicatum. Vue antérieure (Fig. 29), ventrale (Fig. 30) et postérieure (Fig. 31).

\section{Halictus}

(Abb. 36-38). Der Sporn hat einen langen Apex (1/2 der Gesamtlänge) und ein mässig weit ausladendes Velum (entsprechend hoher Spornindex : 3,3). Die dorsale Zähnchenreihe besteht aus sehr kräftigen Zähnen, die ventrale ist schwächer ausgebildet. Die Vorderseite des Stammes ist beschuppt, die Hinterseite (einschliesslich des Velums) hat eine Oberflächenstruktur wie der Stamm von Apis. Die Scharte ist relativ flach (Schartenindex 2,5) und besonders im proximalen Teil beschuppt.

\section{Andrena}

(Abb. 39-41). Der Sporn hat ein sehr schmales, distal und proximal spitz zulaufendes Velum und einen kräftigen Apex, der gut die Hälfte der Spornlänge misst (Spornindex 3,8). Während die dorsale Zahnreihe wohl ausgebildet ist unterscheidet sich die ventrale nur wenig von der Beschuppung der Vorderseite des Stammes, die sie begrenzt. Die Rückseite des Stammes ist schwach beschuppt. Die Scharte ist flacher als bei Apis (Schartenindex 2,1).

\section{Colletes}

(Abb. 42-44). Der Sporn trägt ein distal schmales und proximal etwas breiteres Velum und einen kräftigen Apex ( $1 / 2$ der Gesamtlänge), wodurch sich ein hoher Spornindex $(3,8)$ ergibt. Beide Zahnreihen sind gleichermassen kräftig ausgebildet. Die Oberflächen von Sporn und Velum haben die gleiche Struktur wie bei Apis. Die Scharteninnenfläche, deren proximaler Teil spitze Schuppen trägt ist sehr weit geöffnet bzw. wenig tiế (Schartenindex 2,8). 

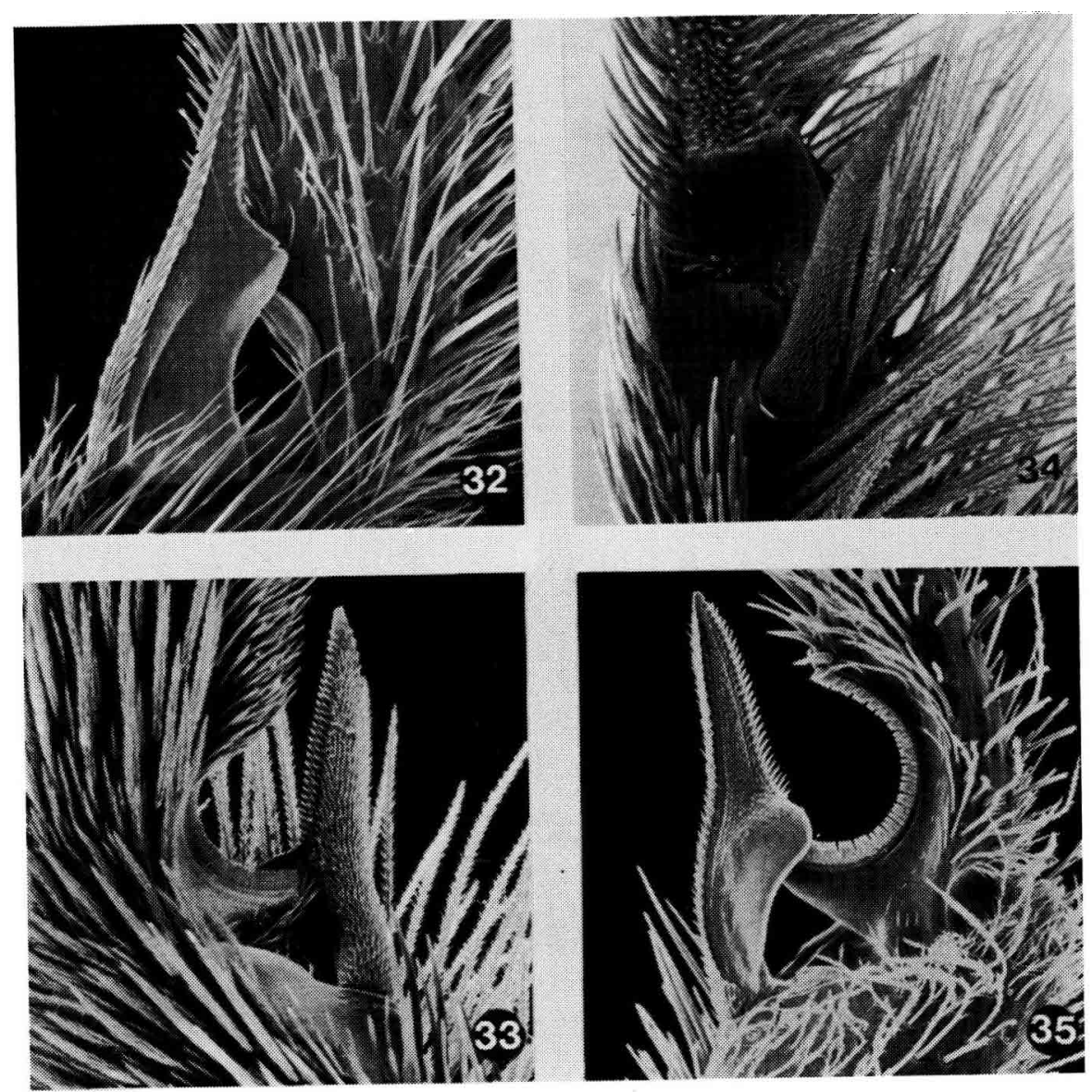

Авв. 32. - Putzapparat einer Osmia fluviventris $f$ ron hinten etwa senkrecht auf das Velum gesehen. Ca. 100 fach.

FIG. 32. - Appareil de nettoyage d'une $f$ d'Osmia fluviventris. Vue postérieure à peu près perpendiculaire au velum. $100 \times$ environ.

AвB. 33 und 34. - Putzapparat von Dasypoda argentata $q$ von vorne (33) und von ventral (34). Ca. 50 fach.

FIG. 33 et 34. - Appareil de nettoyage d'une de Dasypoda argentata. Vue antérieure (Fig. 33) et ventrale (Fig. 34). $50 \times$ environ.

ABв. 35. - Putzapparat von Melitta leporina $j$ von hinten; Ca. 70 fach. Fig. 35. - Appareil de nettoyage d'un $\hat{o}$ de Melitta leporina.

Vue postérieure. $70 \times$ environ. 


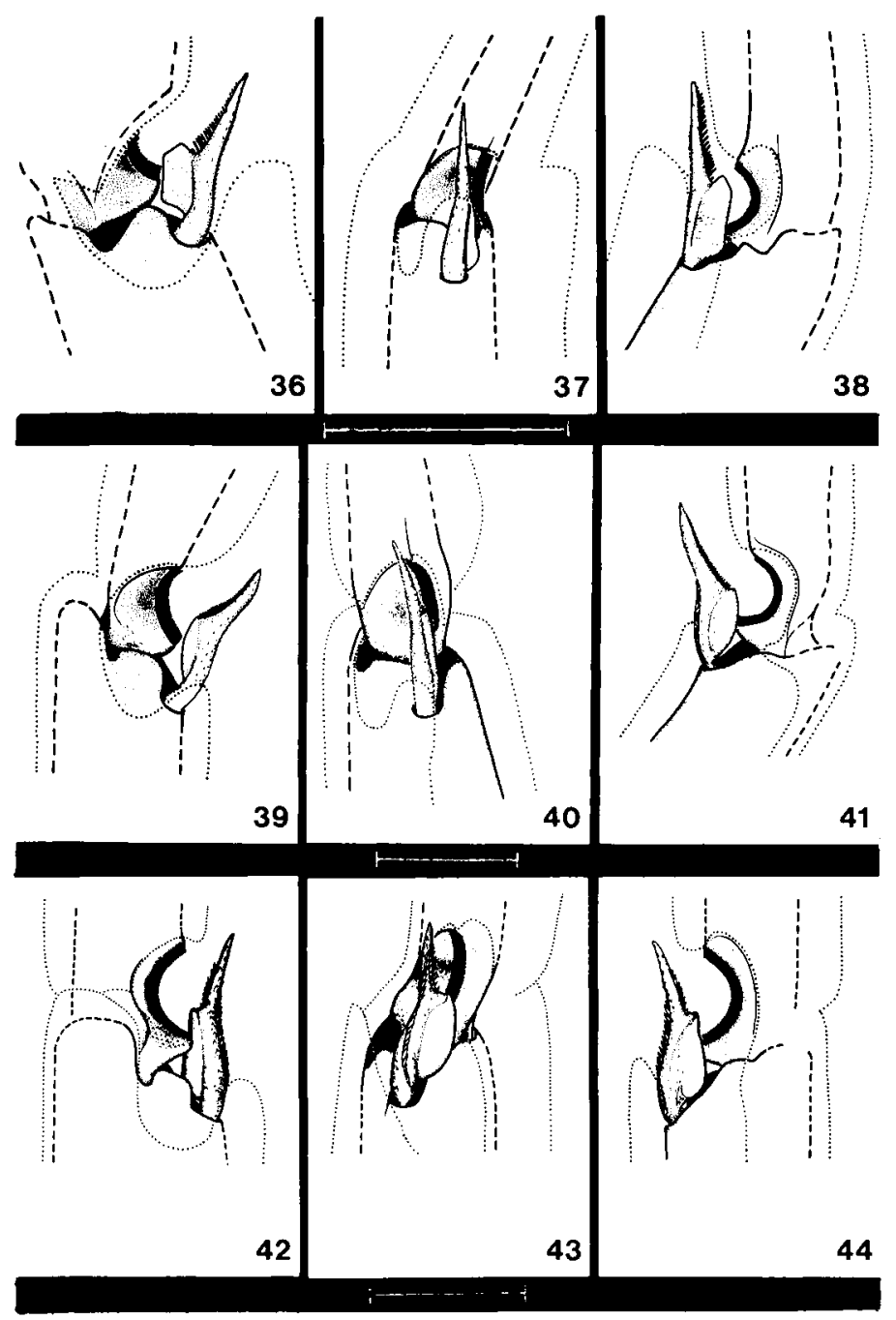

ABB. 36-38. - Putzapparat von Halictus sexcinctus $q$ von vorne (36), von ventral (37) und von hinten (38).

FIG. 36-38. - Appareil de nettoyage d'une $f$ d'Halictus sexcintus. Vue antérieure (Fig. 36), ventrale (Fig. 37) et postérieure (Fig. 38).

Aв8. 39-41. - Putzapparat einer Andrena ovulata 9 von vorne (39), von ventral (40) und von hinten (41).

Fig. 39-41. - Appareil de nettoyage d'une \& d'Andrena ovulata. Vue antérieure (Fig. 39), ventrale (Fig. 40) et postérieure (Fig. 4I).

ABB. 42-44. - Putzapparat von Colletes succinctus $q$ von vorne (42), von ventral (43) und von hinten (44).

Fig. 42-44. - Appareil de nettoyage d'une 9 de Colletes succinctus.

Vue antérieure (Fig. 42), ventrale (Fig. 43) et postérieure (Fig. 44). 


\section{DISKUSSION}

\section{Apidae}

Die vorliegende Beschreibung des Putzapparates von Apis mellifica (Seite 116118 ) bestätigt die älteren Untersuchungen und fügt weicere Details hinzu. Als besonders variabel erwies sich die Oberflächenstruktur des Spornes. Im übrigen ist der Habitus der Putzapparate stets gleich; erst durch den Vergleich der Indices können wir zum Teil einen Unterschied zwischen den drei Morphen erkennen (Tab. 1). Ob hier ein Zusam-

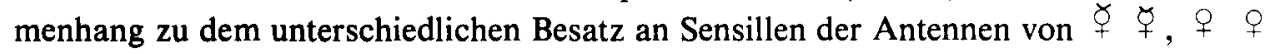
und $\hat{\sigma} \delta$ besteht, erscheint zwar aufgrund des insgesamt sehr ähnlichen Baues der Putzapparate unwahrscheinlich, müsste jedoch erst noch untersucht werden.

Bei den Bombini (Bombus und Psithyrus) fällt die Ähnlichkeit der Putzapparate der verschiedenen Arten auf. Auch die von GenNerich (1922) beschriebenen Putzsporne von Bombus pratorum L. und Psithyrus vestalis FouRCR. - sowie die Zeichnung von ALFORD (1975) - entsprechen im wesentlichen unseren Untersuchungen. GENNERICH erwähnt jedoch nur die äussere (ventrale) Zahnreihe, möglicherweise weil er die zweite mit seinen Hilfsmitteln nicht sehen konnte. Sehr wenig informativ erscheint uns die Zeichnung eines Putzapparates von Bombus lapidarius von WAGNER (1959): In ihr kann man weder Vela noch Zahnreihen erkennen.

Meliponini : Der von GeNNERICH (1922) untersuchte Sporn von Trigona amalthea OL. $q$ ist dem oben beschriebenen Sporn von Melipona scutellaris sehr ähnlich.

Zusammenfassend gilt demnach für die Putzapparate der Apidae (Abb. 45) : Bei Apis, Euglossa, Bombus und Psithyrus trägt der Putzsporn zwei Vela. Bei Melipona und Trigona kommt wie bei allen anderen untersuchten Bienen nur ein nach hinten gerichtetes Velum vor. Die beiden Zahnreihen am Sporn fehlen nur bei Apis und Melipona (und nach GeNNERICH 1922 bei Trigona).

\section{Anthophoridae}

Von der ausserordentlich artenreichen und mannigfaltigen Familie der Anthophoridae gibt es ausser den von uns untersuchten Putzapparaten (von Xylocopa und Anthophora) nur die Beschreibungen der Putzapparate von Eucera difficilis PER. o und von Xylocopa latipes DruRY $\hat{o}$ und $q$ (GenNerich 1922). Wegen der geringen Zahl der untersuchten Arten kann man für die Anthophoridae nicht viel mehr aussagen, als dass sehr verschiedene Putzapparate auftreten. Auch innerhalb der Gattung Xylocopa fanden wir mehrere deutlich verschiedene Putzapparate. Bei $X$. violacea (und bei $X$. latipes, GENNERICH 1922) unterscheiden sich zudem die Sporne von $\delta$ und $q$ (Geschlechtsdimorphismus). 


\section{Megachilidae}

Neben den von uns untersuchten Putzapparaten bei Megachiliden (Anthidium und Osmia) steht uns die Beschreibung der Putzapparate von Thaumatosoma burmanicum BINGH. कs und Megachile maritima KIRB. of und of (GENNERICH 1922) zur Verfügung. Auch in dieser Familie finden wir sehr verschiedene Putzsporne, denen jedoch (ausser bei Megachile maritima, GeNNerich 1922) eine schuppige Oberflächenstruktur gemeinsam ist.

Besonderes Interesse verdient der Sporn von Osmia. Wie wir im folgenden sehen werden, ist er den Spornen der " niederen " Bienen ähnlich.

\section{Melittidae, Halictidae, Andrenidae und Colletidae}

MiCHENER (1974) stellt in seiner Systematik die " langzungigen " phylogenetisch jüngeren Familien der Apidae, Anthophoridae und Megachilidae (und Fideliidae) den phylogenetisch älteren " kurzzungigen " Familien gegenüber. Interessanterweise haben die Vertreter dieser "kurzzungigen" Familien (der Gattungen Colletes, Andrena, Halictus, Melitta und Dasypoda) jeweils ähnliche Vorderbeinsporne (vergl. Abb. 45) mit folgenden Merkmalen :

1. Das Velum ist stets sehr schmal oder nur wenig ausladend.

2. Der Apex ist stets kräftig und misst etwa die Hälfte der gesamten Spornlänge oder mehr (dies wurde unter den langzungigen Bienen nur bei Xylocopa darwini gemessen).

3. Durch das wenig ausladende Velum und den langen Apex liegt der Spornindex bei den " niederen " Bienen stets hoch (den niedrigsten Spornindex dieser Gruppe hat Halictus mit 3.3). Ähnlich hohe Spornindices fanden wir unter den Vertretern der " höheren" Bienen nur bei Psithyrus rupestris und bei P. sylvestris $(2,9)$ sowie bei Osmia $(3,0)$.

4. Schliesslich zeigen die "niederen " Bienen ausser Colletes eine kräftige, meist spitz-dachziegelartige Beschuppung des Stammes, insbesondere seiner Vorderseite. Bei den " höheren" Bienen weisen nur die untersuchten Megachiliden sowie zum Teil Anthophora eine ähnliche Beschuppung auf.

Auch in den Beschreibungen bzw. Zeichnungen der Putzapparate folgender Arten lässt sich der oben beschriebene Typ erkennen : Halictidae : Pseudaugochloropsis graminea + (EICKWORT 1969), Sphecodes spec. (WEBER 1933), Halictoides calochorti CKLl. (SNodgrass 1955), Halictus calceatus SCop. O (GenNerich 1922); Andrenidae : Andrena spec. oै, Panurgus calcaratus Scop. + (GenNerich 1922), Nomada marshamella KIRBY (DAHL 1884); Colletidae: Prosopis annulata L. \& (GeNNERICH 1922); (Wir fanden keine Beschreibung oder Zeichnung eines Putzapparates einer Melittide).

Einen deutlich anderen Putzapparat bei "kurzzungigen" Bienen fanden wir lediglich bei GENNERICH (1922) für Meliturga clavicornis LATR. $q$ und $\vec{j}$, sowie in der 


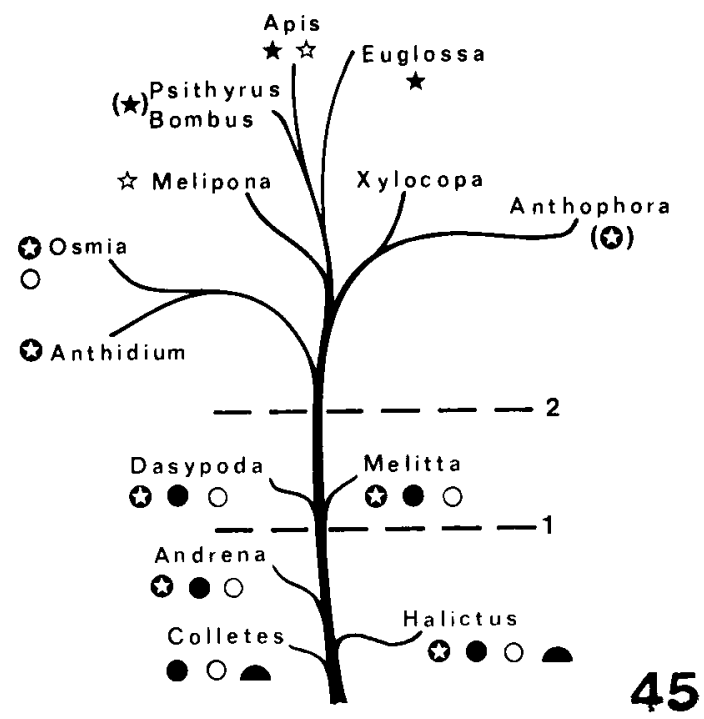

AвB. 45. - Dendrogramm der untersuchten Bienengattungen (nach MICHENER 1974 und W ARNCKE 1977) und wesentliche Merkmale ihrer Putzapparate.

FIG. 45. - Dendrogamme des genres d'abeilles étudiés (d'après MiCHENER 1974 et WARNCKE 1977) et caractéristiques essentielles de leur appareil de nettoyage.

1 Trennung der Apoidea in "Andrenidae " und “Apidae " nach Warncke (1977).

Séparation des Apoïdes en "Andrenidae » et "Apidae " d'après WARNCKe (1977).

2 Trennung der Apoidea in "kurzzungige" und "langzungige" Bienen nach MiCheNer (1974).

Séparation des Apoïdes en abeilles à langue courte et abeilles à langue longue d’après MichENer (1974).

- Spornindex $\geqslant 3,3$.

Indice de l'éperon $\geqslant 3,3$.

Schartenindex $\geqslant 2,5$.

Indice de la brèche $\geqslant 2,5$.

- Vorderseite des Stammes mit spitzen, sich dachziegelartig überdeckenden Schuppen.

Face de l'éperon avec des écailles pointues se recouvrant comme des tuiles.

Länge des Apex ca. 1/2 der Gesamtlänge des Spornes.

Longueur de l'apex, environ la moitié de la longueur totale de l'éperon.

$\star$ Zwei Vela vorhanden.

Présence de 2 velums.

is Keine Zahnreihen am Sporn.

Pas de rangées de dents sur l'éperon.

0 Das betreffende Merkmal ist nur schwach oder teilweise ausgeprägt.

Le caractère en question n'est que faiblement ou partiellement marqué. 
Zeichnung des Putzapparates von Colletes daviesamus von WAGNER (1959). In ihr ist kein Velum zu erkennen, sondern nur ein spangenförmiger, dorsad gebogener, stumpf endigender Sporn. Diese Zeichnung erscheint uns jedoch wenig vertrauenswürdig, da sie keine Details enthält, und zudem die Abbildung vom Putzapparat einer Hummel mit unseren Ergebnissen nicht übereinstimmt (vergl. S. 125).

\section{Hypothetische Phylogenie des Putzapparates der Apoidea}

Aus den vorangegangenen Ausführungen kann man folgern, dass ein hoher Spornindex, ein langer Apex, ein beschuppter Stamm, das Vorhandensein zweier Zahnreihen und eine schuppige Oberfläche des Spornes plesiomorphe Merkmale sind (Abb. 45). Da die Apoidea mit grosser Wahrscheinlichkeit von Sphecoidea-ähnlichen Vorfahren abstammen, ist das Auftreten dieser Merkmale bei Putzapparaten von Sphecoidea (z. B. bei Mellinus arvensis und Gorytes campestris GeNNERICH 1922) eine weitere Stütze unserer Theorie (siehe auch MiCHENER 1944, Seite 227).

Aus der Arbeit von GENNERICH (1922) geht ausserdem hervor, dass ursprüngliche Hymenopteren meist nur eine flache Putzscharte haben. Hieraus kann man schliessen, dass ein hoher Schartenindex plesiomorph für Hymenopteren und damit vermutlich auch für Apoidea ist. Die meisten Apoidea haben eine etwa halbkreisförmige Scharte (mit Spornindices zwischen 1,5 und 2,0), manche aber haben deutlich tiefere Scharten (Psithyrus lucorum, Euglossa spec. und z. T. Apis mellifica 单) mit einem Index von nur 1,2. Schliesslich haben Colletes succinctus und Halictus sexcinctus - als " niedere " Bienen - eine ausnehmend flache Scharte mit einem Index von 2,5 bzw. 2,8.

Ein hypothetischer ursprünglicher Putzapparat, aus dem sich die Putzapparate der rezenten Bienen entwickelt haben könnten, wäre also durch folgende Merkmale zu kennzeichnen :

1. eine relativ flache Scharte;

2. ein langer Apex;

3. ein - und nur ein - schmales Velum;

4. zwei Zahnreihen am Sporn;

5. schuppige Oberflächenstruktur des Spornes.

Innerhalb der niederen "kurzzungigen " Bienen wird dieser Bauplan nur wenig, hauptsächlich durch tiefere Scharten abgewandelt. Es ist uns in dieser Gruppe nur eine Art (Melitturga clavicornis, GeNNERICH 1922) bekannt, die einen deutlich anderen Putzapparat hat (unter Umständen auch Colletes daviesamus, WAGNER 1959; siehe S. 126). Insgesamt können wir einen Entwicklungstrend feststellen zu tiefen Scharten, breiten Vela und kurzen Apices, und nur bei den im System am höchsten stehenden Apidae finden wir teilweise einen Verlust der Zahnreihen und ein zweites (vorderes) Velum.

Während GenNerich (1922) betont, dass der Bau der Putzapparate bei Bienen keinerlei Zusammenhang mit ihrem Verwandtschaftsgrad zeigt, konnte in der 
vorliegenden Arbeit - trotz Vorbehalten wegen der geringen Zahl der untersuchten Arten - doch gezeigt werden, dass ein einheitlicher Typ im Bau der Sporne bei den " kurzzungigen " Familien vorkommt, während bei den " langzungigen " Familien sehr verschiedene Sporne auftreten. Dies entspricht der von MichENER (1974) formulierten Trennung der Apoidea in die Familiengruppe der "kurzzungigen " Bienen als Rest einer alten Radiation ("... the remnants of an apoid radiation...") und der " langzungigen " Bienen als einer phylogenetisch jüngeren Radiationsgruppe (“... a more recent radiation than that of the short-tongued families... "), und bestätigt sie. Ausserdem könnte die Ähnlichkeit des Spornes von Osmia mit den Spornen der " niederen " Bienen ein Hinweis darauf sein, dass die Megachilidae einen älteren Ast der " langzungigen " Bienen darstellen als die Anthophoridae und Apidae, was ebenfalls dem Stammbaumentwurf von MiCHENER (1974) entspricht.

WarnCKe (1977) teilt die Apoidea in (nur) zwei Familien, die Andrenidae (mit Colletinae, Halictinae und Andreninae) und die Apidae, die die " höheren " Bienen einschliesslich der Melittinae umfasst. Diese Zweiteilung der Apoidea geht zwar nicht konform mit dem Bau der Putzapparate, aber auch in seiner Systematik sind die Melittinae nächst den Andreninae, was auch in seinem Dendrogramm deutlich wird.

WAGNer (1959) schreibt, dass sich der Putzapparat “... bei Formiciden, Vespiden und Apiden durch eine... immer stärkere Krümmung des Spornes... weiterentwickelt ». Eine derartige (dorsade) Krümmung des Spornes fanden wir jedoch bei keiner Biene, ebensowenig bei den Beschreibungen und Zeichnungen anderer Autoren - ausser der von WAGNER selbst (Colletes daviesamus; vergl. S. 126). Ausser den Zeichnungen gibt WAGNER keine weiteren Angaben und Erklärungen zu seiner Aussage.

Eingegangen im Februar 1980.

\section{DANKSAGUNG}

Wir danken Herrn Professor Wetzstein, der uns die Benutzung des Rasterelektronenmikroskops im Anatomischen Institut der Universität München ermöglichte.

Die Zoologische Staatssammlung München stellte uns den grössten. Teil der untersuchten Bienen zur Verfügung.

\section{RÉSUMÉ}

L'appareil de nettoyage de 28 espèces provenant des 7 familles les plus importantes des Apoïdes a été étudié au microscope électronique à balayage et décrit.

L'appareil de nettoyage se trouve sur la face ventrale à proximité de l'articulation tibia-basitarse; il est constitue d'une anfractuosité dans la partie la plus proche du basitarse (" brèche ") et d"un éperon articulé. La brèche est limitée à l'arrière par un peigne et plus ou moins largement ouverte. La surface interne de la brèche est en général totalement lisse.

L'éperon porte à sa base un lambeau cutané dirigé vers l'arrière ("velum "). Seuls Apis, Euglossa, Bombus et Psithyrus possèdent un second velum, plus petit et dirigé vers l'avant. Sauf chez Melipona et 
Apis l'éperon porte 2 rangées de dents. La surface de la base de l'éperon est plus ou moins épaisse, avec une structure généralement squameuse.

Les abeilles des familles Colletidae, Andrenidae, Halictidae et Melittidae ont des éperons semblables qui se caractérisent en général par un long apex, un velum étroit et peu proéminent et une structure superficielle fortement squameuse. Ces caractères sont considérés comme primitifs pour les abeilles et correspondent en gros au système formulé par MiCHENER (1974), avec sa division des Apoïdes en 2 groupes de familles.

\section{LITERATUR}

Alford D. V., 1975. - Bumblebees. Davis Polynter, London.

Beeken W., 1934. - Über die Putz- und Säuberungshandlungen der Honigbiene (Apis mellifica). Arch Bienenk. 15, 231-235.

CANestrini und Berlese, 1880. - La Stregghia degli Imenotteri. Atti. Soc. Veneto-Trentina, Padua 7, 53 73 (übernommen aus GENNERICH 1922).

DAHL. F., 1884. - Beiträge zur Kenntnis des Baues und der Funktion der Insektenbeine; Diss. Univ. Kiel.

DE GEeR, 1752/1776. - Memoires pur servir à l'histoire des Insectes. Tome II; (übernommen aus DAHL 1884).

EIckwort G. C., 1969. - A Comparative Morphological Study and a Generic Revision of the Augochlorine Bees (Hym. Halictidae). The Unil. of Kansas Sci. Bull. 48, 325-524.

GENNERICH J., 1922. - Morphologische und biologische Untersuchungen der Putzapparate der Hymenopteren. Arch. f. Naturgeschichte A 88, 1-63.

KIRBY W., 1802. - Monographia apum Angliae. I; Ipswich.

Michener C. D., 1944. - Comparative External Morphology, Pylogeny, and a Classification of the Bees (Hym.). Bull. of the Am. Mus. of the Nat. Hist. 82, 151-326.

MiChENer C. D., 1974. - The Social Behavior of Bees, a Comparative Study. Univ. press, Cambridge, Mass.

Pössinger H., 1948. - Putzmethoden der Rhynchoten. Diss. Univ. München.

Regenfuss H., 1975. - Die Antennen-Putzeinrichtungen der Adephaga (Col.), parallele evolutive Vervollkommnung einer komplexen Struktur. Z. zool. Syst. Evolut.forsch. 13, 278-299.

SNodgrass R. E., 1956. - Anatomy of the Honey Bee. New York.

WaGner M., 1959. - Die Putzhandlungen der Hymenopteren. Diss. Univ. München.

WARNCKE K., 1977. - Ideen zum natürlichen System der Bienen. Mitt. Münch. Ent. Ges. 67, 39-63.

WEBER H., 1933. - Lehrbuch der Entomologie. Jena. 